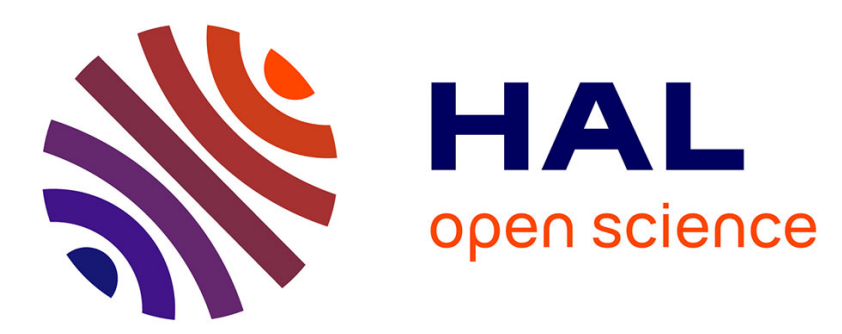

\title{
Texture based image retrieval and classification of very high resolution maritime pine forest images
}

Olivier Regniers, Jean-Pierre da Costa, Gilbert Grenier, Christian Germain, Lionel Bombrun

\section{To cite this version:}

Olivier Regniers, Jean-Pierre da Costa, Gilbert Grenier, Christian Germain, Lionel Bombrun. Texture based image retrieval and classification of very high resolution maritime pine forest images. IEEE International Geoscience and Remote Sensing Symposium, Jul 2013, Melbourne, Australia. pp.40384041. hal-00878727

\section{HAL Id: hal-00878727 https://hal.science/hal-00878727}

Submitted on 30 Oct 2013

HAL is a multi-disciplinary open access archive for the deposit and dissemination of scientific research documents, whether they are published or not. The documents may come from teaching and research institutions in France or abroad, or from public or private research centers.
L'archive ouverte pluridisciplinaire HAL, est destinée au dépôt et à la diffusion de documents scientifiques de niveau recherche, publiés ou non, émanant des établissements d'enseignement et de recherche français ou étrangers, des laboratoires publics ou privés. 


\title{
TEXTURE BASED IMAGE RETRIEVAL AND CLASSIFICATION OF VERY HIGH RESOLUTION MARITIME PINE FOREST IMAGES
}

\author{
O. Regniers, J.-P. Da Costa, G. Grenier, C. Germain, L. Bombrun, \\ IMS Lab., UMR5218 CNRS, Université de Bordeaux, 351 Cours de la Libération, \\ 33405 Talence CEDEX, France \\ olivier.regniers@ims-bordeaux.fr
}

\begin{abstract}
Textural analysis can bring valuable information in the classification or the segmentation process of land covers displaying regular patterns in very high resolution remotely sensed images. In this study, we investigate how features extracted by multivariate modeling of the local spatial dependence in the wavelet domain can efficiently capture the textural content of maritime pine forest images in comparison with a commonly used texture analysis approach, the GLCM. To evaluate the performances of the tested methods, we used a content based image retrieval framework and created a database of image patches representing different development stages of the forest stands. Results show that multivariate models display higher retrieval rates than GLCM-based methods with yet a higher sensitivity to the dominant orientation in anisotropic textures. These observations open up new perspectives in the use of multivariate modeling for textural features extraction in very high resolution image classification.
\end{abstract}

Index Terms - textural feature, multivariate models, GLCM, very high resolution, forest.

\section{INTRODUCTION}

Submetric images produced by the new generation of Very High Resolution (VHR) airborne and spaceborne sensors (e.g. PLEIADES) enable to capture the geometric aspect of small objects which are only observable through their spectral properties at lower resolution. It is for example the case for vegetation rows in vineyards, orchards and young trees stands. The detection and analysis of such land covers can thereby benefit from the use of object-oriented techniques rather than more traditional pixel-based approaches. Many studies have addressed this challenge by considering the planting patterns in the landscape as textures and by computing statistical features on these textures to improve classification or segmentation results on VHR remotely sensed images. In these studies, common tools to characterize textures are Grey Level Cooccurrence Matrices
(GLCM) [1-2], variograms [3], Gabor filters [4-5], wavelet representations [6-7], etc. These approaches all lead to a small-sized representation of the textural content of an image since a few descriptors --- generally referred to as textural signature --- are extracted.

In the wavelet domain, Do \& Vetterli [8] suggested the use of probabilistic models to describe the wavelet coefficients. This technique consisted in modeling the distribution of subbands coefficients with a Probability Density Function (PDF) whose fitting parameters could be further used as a textural signature. More recently, other works investigated the use of multivariate models to express the joint distribution of wavelet coefficients, such as the multivariate Gaussian distribution, Spherically Invariant Random Vectors (SIRVs) [9] and copula based models [10].

In this paper, we focus on the textural analysis of a database of maritime pine forest image patches in a content base image retrieval framework. In particular, we investigate how multivariate models applied in the wavelet domain can improve the image retrieval rate in comparison with other textural analysis approaches such as GLCMs and waveletbased GLCMs.

The paper is structured as follows. Section 2 introduces the texture based image retrieval framework and the multivariate modeling of wavelet coefficients. Section 3 describes the experimentation context. Some texture based classification results are presented in Section 4 on very high resolution maritime pine forest images. Conclusions are finally reported in Section 5.

\section{TEXTURE ANALYSIS}

Texture based image retrieval refers to the automatic retrieval of images from a database based on a set of textural features. The process operates in two steps. The first one is the feature extraction step. Each patch of the database is represented by a finite set of descriptors called features. The second step, called similarity measurement, consists in measuring the similarity between two images based on the set of textural features. 


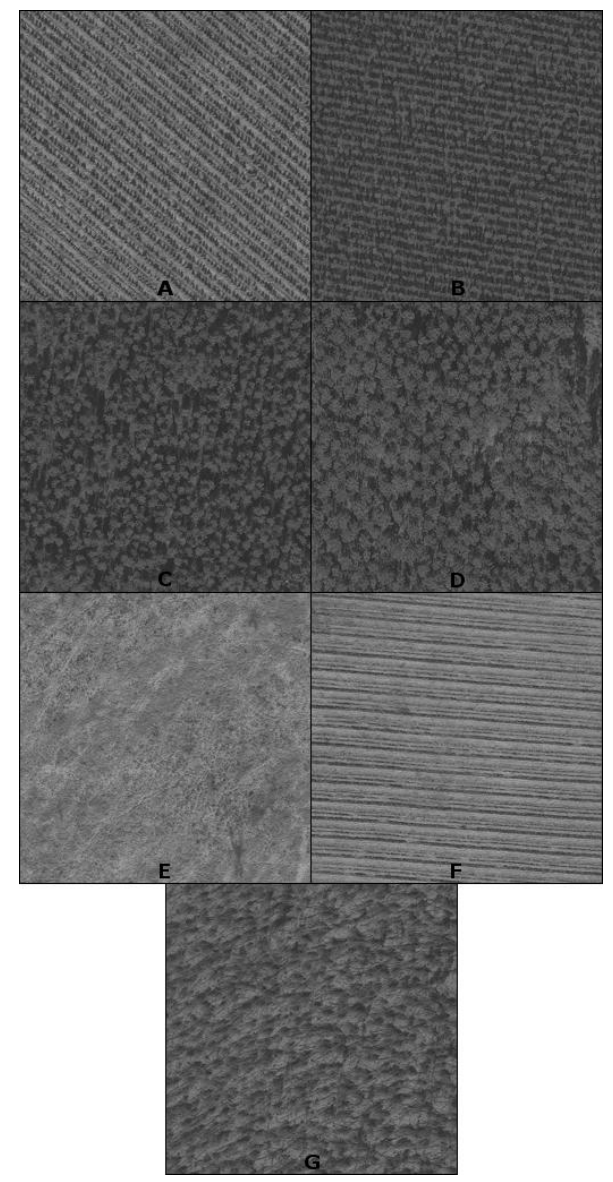

Fig 1: Forest texture classes, (A) forest age class 1, (B) forest age class 2, (C) forest age class 3, (D) forest age class $4,(\mathrm{E})$ clear-cuts, $(\mathrm{F})$ regenerating clear-cuts and

(G) windfall.

In this paper, three techniques are considered in the textural feature extraction step: Grey Level Co-occurrence Matrices (GLCM), Wavelet Co-occurrence Features (WCF) and multivariate stochastic models.

\subsection{GLCM}

The use of the GLCM requires the identification of two control parameters, i.e. the distance and the orientation between the pairs of sites. Given the very high spatial resolution context, the inter-row distance and the multioriented aspect of the vegetation rows, pairs of sites at a distance of 2 pixels in four directions $\left(0^{\circ}, 45^{\circ}, 90^{\circ}\right.$ and $135^{\circ}$ ) are considered for the computation of the matrices. Then, in order to select the right set of textural features, a set of Haralick's second order statistics [11] to which the mean and the variance of grey levels are added, is first derived from the GLCMs. Because many of these features can be redundant due to their high correlation, a further step using a correlation matrix and a Principal Component
Analysis was employed to identify the most useful features. As a result, a subset of four descriptors, homogeneity, entropy, correlation and the mean grey level, were selected. For each image patch of the database, a feature vector containing the four descriptors averaged on the four directions is obtained. Similarity measures based on the Mahalanobis distance are then computed between patches vectors.

\subsection{WCF}

As proposed in [12], GLCMs are computed on each wavelet detail subband using an orthogonal wavelet transform with Daubechies filter $\mathrm{db} 4$ and two scales of decomposition. The original image being down-sampled at each scale of the decomposition, a distance between the pairs of sites of 1 pixel instead of 2 is chosen. As previously, the four GLCM features --- homogeneity, entropy, correlation and mean --- are calculated and averaged on four directions. The resulting feature vectors consist in the concatenation of the four descriptors obtained for each detail subband. A Mahalanobis distance is calculated between the vectors of each image patch to evaluate the similarity.

\subsection{Multivariate Models}

First, an orthogonal wavelet transform with Daubechies filter $\mathrm{db} 4$ and two scales of decomposition is applied on each image patch. For each wavelet subband, $3 \times 3$ neighborhoods are considered. These neighborhoods are regarded as empirical observations of the local spatial dependence of subband wavelet coefficients. The distribution of these observations is modeled using multivariate PDFs whose parameters are estimated according to the maximum likelihood principle. The parameters used on each subband in this fitting procedure constitute the textural signature of the subimage. Several multivariate models are analyzed in this study: the multivariate Gaussian, the Spherically Invariant Random Vector (SIRV) model with a deterministic multiplier (SIRV-Gaussian), the SIRV model with a G0 distribution [9].

Concerning the similarity measure between two feature vectors, an intrinsic distance should be considered. Here, the Rao geodesic distance is chosen since a close form expression exists for the SIRV-Gaussian model. For the G0 distribution, an approximation of the geodesic distance can be done when assuming the geodesic coordinate functions as straight lines. More information concerning the implementation of those models can be found in [9]. 
Table 1: Image retrieval performances $(\%)$

multi-orientation DB

\begin{tabular}{l|cc}
\multicolumn{1}{c}{ Methods } & ARR & kNN* \\
\hline GLCM & $38.2 \pm 0.9$ & $60.5 \pm 2.0$ \\
WCF & $30.9 \pm 0.7$ & $54.5 \pm 2.6$ \\
Gaussian SCM & $34.6 \pm 1.0$ & $62.5 \pm 1.7$ \\
SIRV - Gaussian & $\mathbf{4 0 . 0} \pm 1.0$ & $\mathbf{7 3 . 0} \pm 1.7$ \\
SIRV - G0 & $\mathbf{3 9 . 6} \pm 0.9$ & $\mathbf{7 0 . 5} \pm 1.5$ \\
Gaussian Copula & $36.5 \pm 1.0$ & $66.8 \pm 1.2$ \\
\hline \multicolumn{3}{c}{ mono-orientation DB } \\
Methods & ARR & kNN* \\
\hline GLCM & $38.3 \pm 1.4$ & $59.3 \pm 2.3$ \\
WCF & $32.0 \pm 0.7$ & $57.8 \pm 2.6$ \\
Gaussian SCM & $38.6 \pm 1.0$ & $71.4 \pm 1.7$ \\
SIRV - Gaussian & $\mathbf{4 8 . 5} \pm 1.1$ & $\mathbf{7 6 . 3} \pm 1.9$ \\
SIRV - G0 & $\mathbf{4 6 . 3} \pm 1.2$ & $\mathbf{7 6 . 0} \pm 2.0$ \\
Gaussian Copula & $39.1 \pm 1.0$ & $69.3 \pm 1.8$ \\
\hline \multicolumn{2}{c}{$*$ k = 10 - Iterations $=25-$ Patches per class = 50 }
\end{tabular}

\section{EXPERIMENTATION}

We used panchromatic VHR airborne images $(0.5 \mathrm{~m}$ resolution) acquired in February 2009 in the Landes de Gascogne forest, South West of France, where the dominant vegetation consists of managed maritime pine (Pinus pinaster) stands. The forest structure is heterogeneous with stand ages ranging from 0 (clear cuts) to approximately 50 years. These images were acquired a few weeks after a storm and significant windfall damages can be observed throughout the landscape.

A large database of texture patches was created by selecting homogeneous regions of $256 \times 256$ pixels representative of 7 identified classes of interest: 4 age classes defined by the average crown diameter in the area, one class representing windfall damages and two transitional classes corresponding to clear cut stages, namely clear cuts and regenerating clear cuts (Fig.1).

A second database was produced in order to assess the sensitivity of the tested models to orientation. Unlike in the first database where the native dominant orientation of the stands is preserved (multi-orientation DB), each patch displaying an anisotropic texture is rotated so that its main orientation is forced to $0^{\circ}$ (mono-orientation DB).

For each method, a feature vector containing the signature is obtained for each texture image. Based on these feature vectors, a similarity measure is computed between the signatures of each patch of the database resulting in an $N x N$ distance matrix where $N$ is the total number of images in the database.

An equal number of images is extracted from each class of the database by random selection. Each image patch is then considered as a query image. The top matches are
Table 2: Per-class Average Retrieval Rate (\%)

\begin{tabular}{|c|c|c|c|c|}
\hline \multicolumn{5}{|c|}{$\underline{\text { multi-orientation DB }}$} \\
\hline & GLCM & $\begin{array}{c}\text { SIRV - } \\
\text { Gaussian }\end{array}$ & $\begin{array}{c}\text { SIRV - } \\
\text { G0 }\end{array}$ & $\begin{array}{c}\text { Gaussian } \\
\text { Copula }\end{array}$ \\
\hline Forest 1 & 32.0 & 16.5 & 23.8 & 31.1 \\
\hline Forest 2 & 43.1 & 30.9 & 33.2 & 30.8 \\
\hline Forest 3 & 33.0 & 47.6 & 44.5 & 39.2 \\
\hline Forest 4 & 37.2 & 59.2 & 56.4 & 47.2 \\
\hline Windfall & 46.4 & 52.5 & 55.3 & 48.3 \\
\hline $\mathrm{CC}$ & 41.3 & 54.1 & 44.0 & 36.4 \\
\hline $\mathrm{rCC}$ & 34.1 & 19.3 & 20.3 & 22.8 \\
\hline \multicolumn{5}{|c|}{ mono-orientation DB } \\
\hline & GLCM & $\begin{array}{c}\text { SIRV - } \\
\text { Gaussian }\end{array}$ & $\begin{array}{c}\text { SIRV - } \\
\text { G0 }\end{array}$ & $\begin{array}{c}\text { Gaussian } \\
\text { Copula }\end{array}$ \\
\hline Forest 1 & 32.6 & 44.0 & 47.5 & 44.8 \\
\hline Forest 2 & 42.6 & 48.6 & 46.1 & 34.7 \\
\hline Forest 3 & 33.1 & 39.9 & 40.1 & 37.1 \\
\hline Forest 4 & 37.8 & 60.8 & 58.8 & 49.8 \\
\hline Windfall & 46.1 & 45.0 & 50.7 & 41.8 \\
\hline $\mathrm{CC}$ & 41.7 & 48.8 & 40.3 & 33.6 \\
\hline $\mathrm{rCC}$ & 34.3 & 52.4 & 40.3 & 32.1 \\
\hline
\end{tabular}

extracted from the distance matrix to evaluate the image retrieval performance of each method using two different approaches: (1) the Average Retrieval Rate (ARR) defined as the average percentage of images belonging to the same class as the query in the top $n_{i}$ matches, where $n_{i}$ is the number of patches in the class $i$ of the query and (2) the overall accuracy computed after a $k$ Nearest Neighbor $(\mathrm{kNN})$ classifier. This random selection process is repeated several times. The average performance and its standard deviation are computed.

\section{RESULTS AND DISCUSSION}

The resulting mean performances obtained for 25 iterations and 50 image patches per class (Table 1) display higher retrieval rates for multivariate models than for GLCM-based approaches, especially in the case of SIRV-based models. This tends to show that in this study context, the multiscale multivariate modeling of the spatial dependence of wavelet coefficients enables to capture textures more accurately than second-order statistics such as GLCMs. However, global performances stay low which is most probably due to high intra-class variability and inter-class overlapping.

Results in Table 1 also point out a sensitivity of the tested multivariate models to orientation. Their retrieval performances for the multi-orientation database are indeed lower than for the mono-orientation database, while performances remain similar for both databases in the case of the GLCM method. In other words, multivariate models do not comply with rotation invariant requirements. This is 
also confirmed by per-class performances (Table 2) for the multi-orientation database where GLCM method shows higher performances for typical anisotropic textures (forest age class 1 , forest age class 2 and regenerating clear-cuts) while multivariate models are more efficient in retrieving isotropic textures. Nevertheless, once orientation is compensated as in the mono-orientation database, multivariate models exhibit better performances for all classes.

\section{CONCLUSIONS}

In conclusion, the characterization of textures with multivariate models in the wavelet domain, in particular SIRV-based models, is appealing in the context of forest age classes image retrieval in comparison to more traditional second-order statistics such as GLCMs. These approaches should nevertheless be used with caution in the case of anisotropic textures unless the dominant orientation has been previously corrected. These results suggest interesting perspectives in terms of use of multivariate models in texture based classification of regularly patterned land covers.

\section{ACKNOWLEDGEMENTS}

The authors wish to thank CNES (Centre National d'Etudes Spatiales, Toulouse, France), Aquitaine Regional Council, AgriMIP Sud Ouest and Aerospace Valley Clusters for the financial support of this work. The authors also gratefully acknowledge INRA (Institut National de la Recherche Agronomique, Villenave d'Ornon, France) and particularly the EPHYSE research unit for providing the VHR airborne images used in this study.

\section{REFERENCES}

[1] Franklin S.E., Wulder M.A., Gerylo G.R., "Texture analysis of IKONOS panchromatic data for Douglas-fir forest age class separability in British Columbia", Int. J. Remote Sensing, 22, 2627-2632, 2001.

[2] Kayitakire F., Hamel C., Defourny P., "Retrieving forest structure variables based on image texture analysis and IKONOS-2 imagery", Remote Sens. Environ., 102, 390-401, 2006.

[3] Trias-Sanz R., "Texture orientation and period estimator for discriminating between forests, orchards, vineyards, and tilled fields", IEEE Trans. Geosci. Remote Sens., 44, 2755-2760, 2006.

[4] Delenne C., Rabatel G., Deshayes M., “An automatized frequency analysis for vine plot detection and delineation in remote sensing", IEEE Geosci. Remote Sens. Lett., 5, 341-345, 2008.
[5] Aksoy S., Akçay H.G., Wassenaar T., "Automatic mapping of linear woody vegetation features in agricultural landscapes using very high resolution imagery", IEEE Trans. Geosci. Remote Sens., 48, 511-522, 2010.

[6] Ruiz L.A., Fdez-Sarría A., Recio J. A., "Texture feature extraction for classification of remote sensing data using wavelet decomposition: a comparative study", Int. Arch. Photogram. Rem. Sens. Spatial Inform. Sci., XXXV-B4, 1109-1114, 2004.

[7] Lefebvre A., Corpetti T., Hubert-Moy L., "Segmentation of very high spatial resolution panchromatic images based on wavelets and evidence theory", SPIE Remote Sensing Conf., 7830, 2010.

[8] Do M.N., Vetterli M., "Wavelet-based texture retrieval using generalized Gaussian density and Kullback-Leibler distance", IEEE Trans. Image Process., 11, 146-158, 2002.

[9] Bombrun L, Berthoumieu Y., Lasmar N.-E., Verdoolaege G., "Multivariate texture retrieval using the geodesic distance between elliptically distributed random variables", 18th IEEE Int. Conf. on Image Processing (ICIP), 3637-3640, 2011.

[10] Kwitt, R., Uhl A., “A joint model of complex wavelet coefficients for texture retrieval", 16th IEEE Int. Conf. on Image Processing (ICIP), 1877-1880, 2009.

[11] Haralick R.M., Shanmugam K., Dinstein I., "Textural features for image classification", IEEE Trans. Syst., Man, Cybern., Syst.., 6, 610-621, 1973.

[12] Van de Wouwer G., Scheunders P., Van Dyck D., "Statistical texture characterization from discrete wavelet representations", IEEE Trans. Image Process., 8, 592-598, 1999. 